\title{
A Comparative Analysis of Machine Learning Algorithms Used For Training in Face Recognition
}

\author{
M. Rajalakshmi ${ }^{1}$, S Srividhya ${ }^{2}$ \\ ${ }^{1}$ Assistant Professor, Department of information technology, SRM Institute of Science and Technology, Tamil, \\ rajalakm2@srmist.edu.in \\ ${ }^{2}$ Assistant Professor, Department of information technology, SRM Institute of Science and Technology, Tamil \\ Nadu, India, 603203, Country, srividhs1@ srmist.edu.in
}

\begin{abstract}
To human identity, the face is the most important one that best identifies an individual. Face recognition is very common in today's modern world, and is widely used for much surveillance, safety, retail, tourism, healthcare, and hospitality applications. In the world of facial detection there have been several developments over the years. In this manuscript, the proposed algorithms are likened to the $\mathrm{K}$ nearest neighbor, Support Vector Machine, Decision Tree Classifier, Random Forest, Naïve Bayes algorithms. Despite several benefits it provides, the precision of facial recognition may be further increased in cases where the conditions for image processing are not so good and where the photographs of a face are taken many years apart. The accuracy of facial recognition varies according to the technique used and the circumstances under which they are evaluated. The objective of this manuscript is to compared with different machine learning algorithms used for classification depending upon accuracy, precision, sensitivity, specificity. The outcome reveals that all the other algorithms except SVM and KNN fell below 90 per cent in the metrics while the reliability of the test set tends to be reasonably successful for all the algorithms. It was observed that picture face orientation plays a major role in correct face recognition. Both measurements are decreased by 40 percent without face synchronization of the images and hit 60 percent for SVM and even less for other algorithms.
\end{abstract}

Key words: Convolutional Neural Network, Computer Vision, FaceNet, Face Recognition, Machine Learning Algorithms

\section{INTRODUCTION}

Face recognition is a mode of utilizing technology to identify human faces. Facial recognition system clarifies facial features from an image or video using biometrics. It compares the details of recognized faces database to identify the match. Furthermore, the gap between the eyes, the nose, the lips and the chin, the upper dimensions of the eye sockets, the sides of the lips, the location of the nose and eyes and the area around the check bones are all measured for precision.

The key feature of facial recognition is that it is not invasive and can be performed from a distance, even without the user being aware of the scanning. This sort of thing is needed in high-security areas are banks, government offices and public places such as bus stops, train stations, general meeting places like malls, parks etc. Face recognition devices at these sites are different from other biometric methods that can be used for surveillance purposes such as looking for wanted suspects, alleged terrorists or missing children. Face recognition is a technology which identifies or verifies a person's identity. Face recognition technology is become more popular in the upcoming future. This technology can recognize or verify an individual from the video source in digital image or video frame based on system-trained database. Facenet [1] maps each representation of the face to the Euclidean distance, i.e. that distances corresponding for the similarities of the face. FaceNet reaches 99.6 per cent precision based on the sample Labelled Faces in the Wild (LFW). Based on the connection to this study, the information used in this paper has been extracted [2].

Moreover, it is determined as an application centered on biometric artificial intelligence that can uniquely identify an individual through assessing patterns focused on the individual's facial textures including shape. In recent years, it has broader applications on mobile devices, and is commonly used in other modes of technology, such as robotics, due to its contactless and non-invasive operation. There are already a number of different algorithms for face recognition with various strategies and characteristics involved, and they are still improving. The objective of this manuscript is to compare various Machine Learning (ML) algorithms [3-9], which are used to train the Face Recognition Model.

\section{LITERATURE SURVEY}

In [10], four existing algorithms and a new voting algorithm were applied on multiple datasets, and results were evaluated using ROC CURVE and accuracy of verification. The 
technique used for face recognition using $\mathrm{KNN}$ [1] was common use for identical faces. In [11] KNN, Naïve Bayes comparison, Decision tree was rendered and the machine learning classifiers were constructed using Alex Net Model where accuracy, sensitivity, precision, and specificity were used to calculate overall performance. Using CNN was introduced in [12] face recognition, and its precision has been noticed.

Rathod et al [13] created automatic visitation-take framework depending upon algorithms for face identification along identification. For example, once the camera has been mounted in a classroom, it captures photos; it includes entire students faces present at the class. The facial area of the pupil was then removed and pre-processed for further analysis. This device can be able to identify and recognize each student automatically. The names were imported into an Excel spreadsheet after remembering the identities of the students. In addition, the spoofing of facial recognition was done using an anti spoofing tool, such as the eye twitch detector. Wei et al. [14] tackle, using a digital methodology, the in-class social network creation problem including pedagogical examines. In data processing, a teacher takes several pictures of students in a class and using an image stitching algorithm, these pictures were merged into one single image. Then the website of the course lets the tutor uploading a stitching file. Facial identification, pupil translation, facial recognition approaches have been utilized to classify the names with locations of the pupils. Pupils then $\log$ in to the website and verify their attendance and complete the attendance register, mark each class with their own name. The sitting arrangements can be utilized to create social network at the semester end. Academic success of students with pedagogical study of co-learning process could be built dynamically with the social network statistics.

\section{DATASET USED}

\section{A.Database}

The TUFT dataset consists of over 10,000 separate photos along the range of age 4-70 years and the dataset has many image models: visible, near-infrared, thermal, computerized sketch, etc. The dataset used here consists of RBG image of 113 individuals with 14 images per class which amounts to a total of 1,582 images.

\section{B.Data Augmentation}

The significance of image enhancement in face recognition by adding different transformations to the image is discussed in $[15,16]$. The artefacts in the dataset are improved by the implementation of transformations such as switch, cover, and add noise. Only the testing range, and not the evaluation range, is raised.

\section{METHODOLOGY}

\section{Feature Extraction}

Feature extraction is a method of selecting and /or integrates variables as features, efficiently minimizing the count of data executable, describing the original data set more accurately as well as completely.

\section{FaceNet}

FaceNet model as shown in Figure 1 provides centralized embedding for activities related to facial identification, authentication, clustering. It cartography every facial image to Euclidean distance, i.e. that distance is equivalent to facial similarity, for example, image of individual A would be positioned relative to all other images of individual A than any other person present in the dataset.

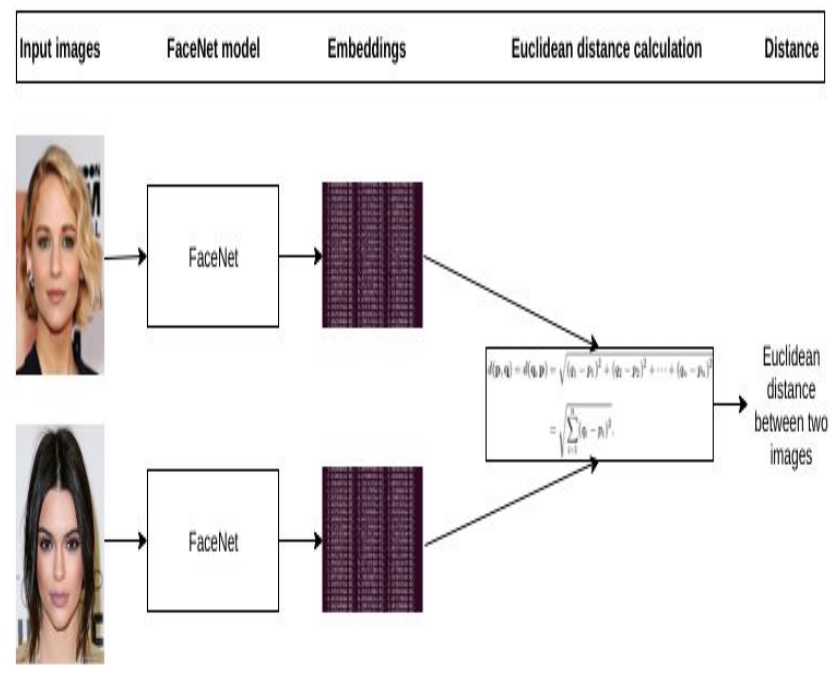

Figure 1: FaceNet Model Architecture

The key differentiation amid FaceNet and other approaches is that it learns mapping from images also generates embedding instead of using some form of bottleneck to recognize or validate function. If the embedding is developed, all the other tasks such as testing, identification etc. can be carried out using normal techniques in that specific area, using this newly generated embedding as the vector of the function. For instance, we can use k-NN for face recognition by using embedding as the feature vector and similarly we can use any clustering technique to cluster the faces together and we just need to identify a threshold value for verification. Face Net uses a deep neural convolution network (CNN).

The network is equipped in such a way that the squared L2 distance between the embedding equals similarity. The photographs used for the training like measured, shaped and cut closely around the region of the face. Their loss role is another significant feature of Face Net. 


$$
L o s s=\sum_{i=1}^{N}\left[\left\|f_{i}^{a}-f_{i}^{p}\right\|_{2}^{2}-\left\|f_{i}^{a}-f_{i}^{n}\right\|_{2}^{2}+\alpha\right]_{+}
$$

It uses triplet loss function as shown in Figure 2 (a) and (b), which can be defined as the distances between anchor image embedding and positive image embedding.

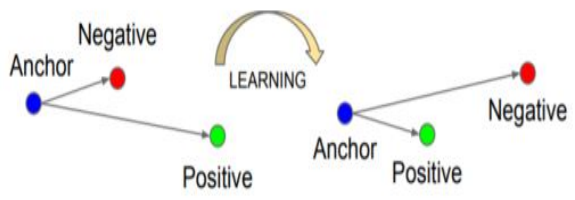

(a)

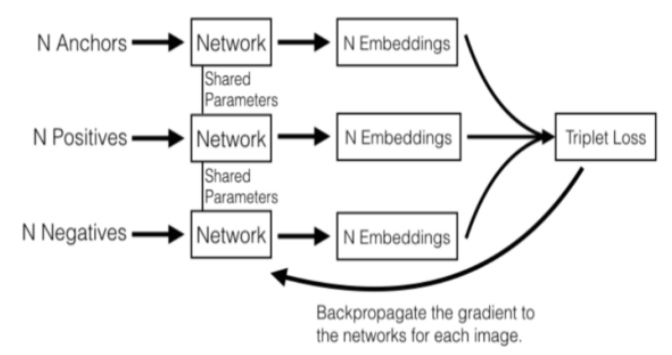

(b)

Figure 2: (a) Triplet Loss (b) Triplet loss training technique.

\section{MACHINE LEARNING ALGORITHMS COMPARED}

\subsection{Support Vector Machine (SVM)}

This is depending upon detecting the hyper-plane concept, which optimal splits the dataset as 2 groups. An SVM model is essentially representation in multi-dimensional space of various groups within a hyper-plane. SVM will generate the hyper-plane iteratively, so the error could be decreased. The objective of SVM has been partition the datasets as groups in order to reach a maximal cumulative hyper-plane (MMH).

\subsection{K Nearest Neighbour (KNN)}

KNN is the straightforward algorithm that saves entire possible events as well as classifies new events in terms of resemblance metric (e.g., distance operations), $\mathrm{K}$ nearest neighbor has been utilized in statistical evaluation with pattern recognition. KNN algorithm is used in supervised learning, to both regressions including classification. KNN approach is a method of grouping of cases based on their similarities with other cases. Events adjacent to both of these are considered neighbors. A significance is allocated to the new data point depending on its closeness to the points within the training collection. The KNN is considered a lazy training algorithm, since there is no advanced process of preparation. KNN does not make any claims on the underlying results. In [17], accuracy was observed to be higher with lower k values, and decreased for higher k values. With large dataset and high dimensions, the KNN algorithm doesn't work well.

The KNN function could be illustrated in the following algorithm:

\section{Algorithm: KNN}

Step 1: The value for $\mathrm{k}$ is selected, where $\mathrm{k}$ is the number of neighbors.

Step 2: Euclidean distance is calculated between k numbers of neighbors.

Step 3: K nearest neighbors is obtained in terms of calculated Euclidean distance.

Step 4: The count of data points is counted at every group.

Step 5: New data points have been allocated for a group based on similarity.

\subsection{Random Forest (RF)}

$\mathrm{RF}$ is the robust machine learning system capable of performing both regression and classification tasks, even performing dimensional reduction processes, handling missing values, outer values and other important data discovery measures. It is ensemble learning approach type, also a weak samples category integrate to create an efficient method. The random classification of forests is based on the classification of tree decisions. RF contains a set of individual decision trees, which function as a collective. Any single tree at the random forests produces class forecast, the default forecast becomes the class with the largest number of votes. One of the main characteristics of Random Forest is the poor intermodal correlation. It helps the trees defend each other from human mistakes. Research explains [18] that Random Forest's output in face recognition is strong as a support classifier for vector machines. The Random Forest algorithm is very highly effective. Random Forest ROC zone is higher than SVM.

\subsection{Naive Bayes algorithm}

This is a classification approach with the assumption of freedom amid Bayes ' theorem based predictors. A Naive Bayes classifier, basically, that adding one specific feature to the class is immaterial for any feature. Eg, if a fruit is red, round with a diameter of 3 inches, it can be termed as apple. Particularly, if the traits depending upon every other or the presence of different traits, all of these fruits individually contribute for possibility of being an apple. Naive Bayes method is easy to configure and very useful for very huge data sets and even extremely advanced classification methods are known to outperform.

\section{Algorithm Naive Bayes}

Step 1: Compute the previous probability of provided class labels

Step 2: Identify Likelihood probability along every attribute to every class

Step 3: Keep the values at Bayes Formula, also compute posterior probability.

Step 4: Since the input belongs to the high probability class, find out that class has the highest probability.

\subsection{Linear Discriminant Analysis (LDA)}

It is the generalized Fisher's linear discrimination, the strategy 
utilized at statistics, pattern identification, machine learning for locating features linear combinations, which categorize or differentiate 2 or more groups of items or events. Linear discriminant analysis methodology standard application includes all the samples to be made available beforehand. However, there are cases where the entire dataset is inaccessible then the input data is interpreted as current. Here, the extraction of the linear discriminant analysis function can upgrade the calculated linear discriminant analysis features through analyzing new models without running the algorithm on the entire data collection. On the other hand, Linear Discriminant Analysis is a supervised algorithm that identifies linear discriminants, which are going to represent certain axes that optimize differentiation between different groups. Classification of facial images using the desired outputs based on Linear Discriminate Analysis was addressed in [19]. For example, as new insights become possible at multi-real-time utilizations like mobile robotics or on-line facial recognition it has been necessary for upgrading the extracted linear discrimination analysis features.
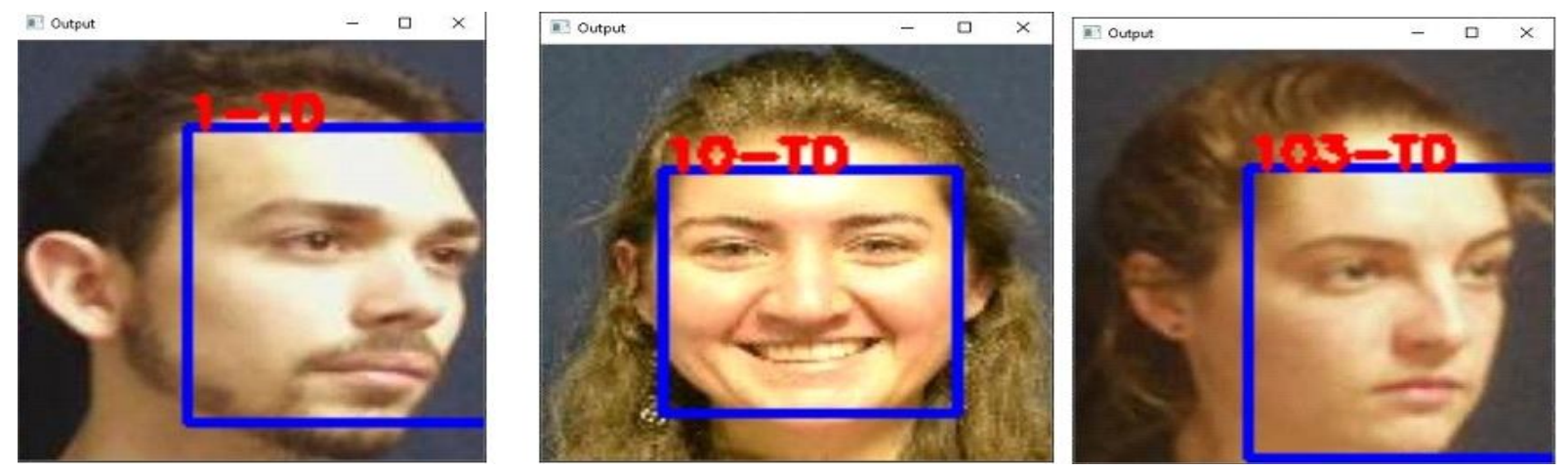

\begin{tabular}{|c|c|c|c|c|c|c|}
\hline S.No & Algorithm & $\begin{array}{c}\text { Type of } \\
\text { validation }\end{array}$ & $\begin{array}{c}\text { Accuracy } \\
\mathbf{x 1 0 0}(\%)\end{array}$ & $\begin{array}{l}\text { Precision } \\
\mathbf{x 1 0 0}(\%)\end{array}$ & $\begin{array}{c}\text { Recall } \\
\mathbf{x 1 0 0}(\%)\end{array}$ & $\begin{array}{l}\text { F1 Score } \\
\text { x100(\%) }\end{array}$ \\
\hline \multirow[b]{2}{*}{1} & \multirow[b]{2}{*}{ SVM } & $\begin{array}{c}\text { K-Fold } \\
\text { Validation }\end{array}$ & 0.917012 & 0.921511 & 0.917012 & 0.917822 \\
\hline & & $\begin{array}{l}\text { Hold Out (test) } \\
\text { set } \\
\text { validation }\end{array}$ & 0.987013 & 0.993506 & 0.987013 & 0.988590 \\
\hline \multirow[b]{2}{*}{2} & \multirow[b]{2}{*}{ KNN } & $\begin{array}{c}\text { K-Fold } \\
\text { Validation }\end{array}$ & 0.906487 & 0.909399 & 0.906487 & 0.906788 \\
\hline & & $\begin{array}{l}\text { Hold Out (test) } \\
\text { set validation }\end{array}$ & 0.990260 & 0.993506 & 0.990260 & 0.990538 \\
\hline \multirow[t]{2}{*}{3} & \multirow{2}{*}{$\begin{array}{l}\text { Random } \\
\text { Forest }\end{array}$} & $\begin{array}{c}\text { K-Fold } \\
\text { Validation }\end{array}$ & 0.888716 & 0.893173 & 0.888716 & 0.888929 \\
\hline & & $\begin{array}{l}\text { Hold Out } \\
\text { (test) set } \\
\text { validation }\end{array}$ & 0.990260 & 0.992695 & 0.990260 & 0.989981 \\
\hline \multirow[t]{2}{*}{4} & \multirow[t]{2}{*}{ Naive Bayes } & $\begin{array}{c}\text { K-Fold } \\
\text { Validation }\end{array}$ & 0.857315 & 0.875133 & 0.857315 & 0.861743 \\
\hline & & $\begin{array}{l}\text { Hold Out (test) } \\
\text { set } \\
\text { validation }\end{array}$ & 0.983766 & 0.984578 & 0.983766 & 0.982004 \\
\hline \multirow[t]{2}{*}{5} & \multirow{2}{*}{$\begin{array}{c}\text { Linear } \\
\text { Discriminant } \\
\text { Analysis }\end{array}$} & $\begin{array}{c}\text { K-Fold } \\
\text { Validation }\end{array}$ & 0.894237 & 0.903968 & 0.894237 & 0.896412 \\
\hline & & $\begin{array}{c}\text { Hold Out } \\
\text { (test) set } \\
\text { validation }\end{array}$ & 0.990260 & 0.993506 & 0.990260 & 0.990291 \\
\hline \multirow[t]{2}{*}{6} & \multirow[t]{2}{*}{$\begin{array}{c}\text { Logistic } \\
\text { Regression }\end{array}$} & $\begin{array}{c}\text { K-Fold } \\
\text { Validation }\end{array}$ & 0.869565 & 0.876000 & 0.869565 & 0.868939 \\
\hline & & $\begin{array}{l}\text { Hold Out (test) } \\
\text { set validation }\end{array}$ & 0.980519 & 0.983225 & 0.980519 & 0.979066 \\
\hline
\end{tabular}




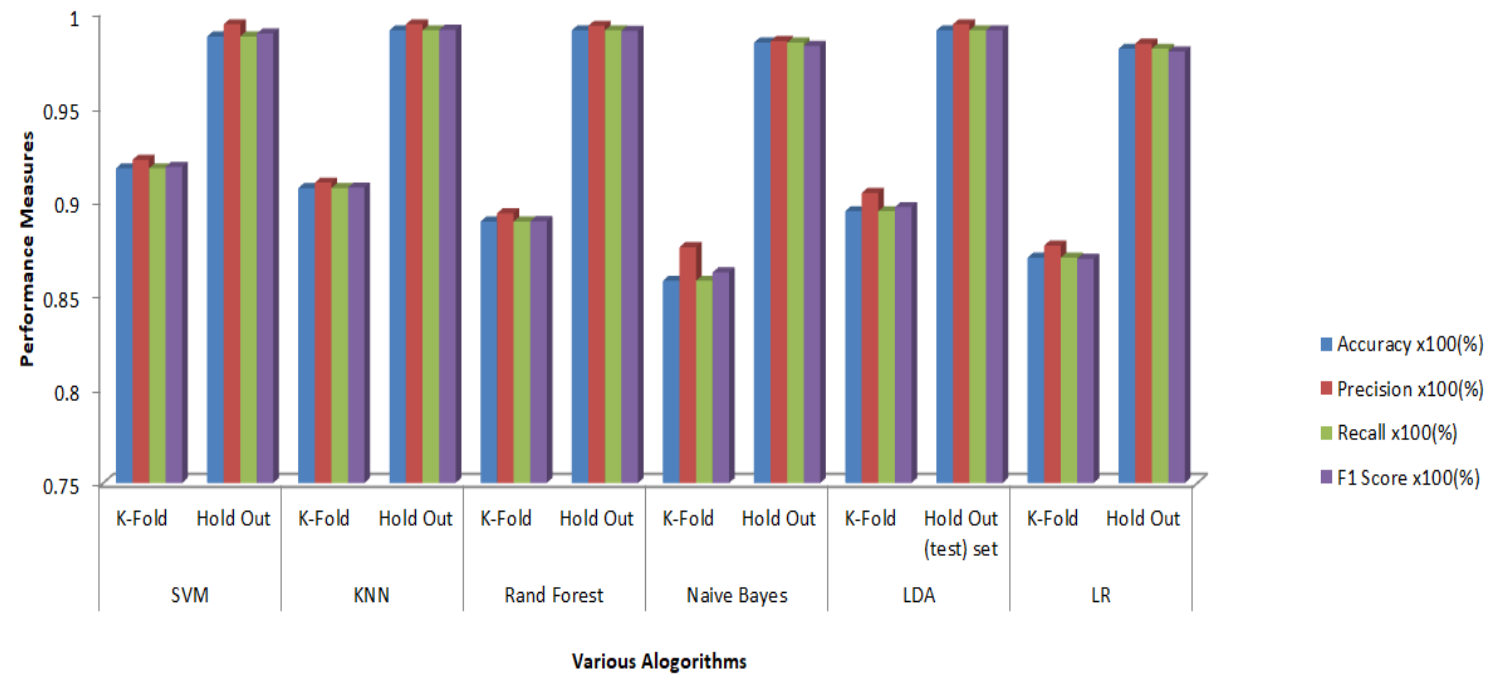

Table 1: Comparative results of different algorithms

An LDA extraction technique capable of upgrading the linear discrimination analysis features through merely analyzing new models has been incremental linear discrimination analysis approach; moreover this principle is fully explored over the past two decades.

\subsection{Logistic Regression}

It is to test the relationship amid the dependence as well as one or more independence variables, by calculating probabilities utilizing their basic logistic method. Then these odds can be converted as binary values to essentially create a prediction. This logistic function task is mostly known as sigmoid function. The Sigmoid-Function represents S-shaped curve that consider any real-value number, also represent the value among 0 and 1 , but not specifically within theirs limitation. Between 0 and 1 these values are converted to 0 or 1 using a classifier to the threshold.

\section{EXPERIMENTAL RESULTS}

The data collection split into the training and test system consists of 1243 (without augmented images) and respectively 340 images. The faces in the images are detected and aligned using openface alignment, which uses Dlib's face detection and alignment [20] and approximately 83 percent of the training images and 93 percent of test images are detected and aligned correctly. The alignment involves aligning the outside eyes and nose in such a way that in all pictures, the positions are at the same spot. The picture being stored the dimension 96x96. The processing image's BlobImage is then fed to the pre-trained model of the openface to remove the 128-d embedding [1].

The $128 \mathrm{~d}$ embedding testing is performed with various algorithms, and the expected value is compared to the real value. The assessment is conducted on both training set and test set. In the case of training collection, $\mathrm{K}$ - fold validation is done to reduce variation. The table 5.1 depicts the results obtained from the experiment and corresponding accuracy, precision, recall for the algorithms. The results involve both hold out validation and $\mathrm{k}$ - fold validation. The results shows that all the other algorithms except SVM and KNN falls less than $90 \%$ in the metrics, although the test set validation seems fairly good for all the algorithms. It was found that the face aligning of the image plays a major role in accurate face recognition. Without face alignment of the images, all metrics are reduced $40 \%$ and reaches $60 \%$ of SVM and much lesser in case of other algorithms.

\section{CONCLUSION}

When face recognition was achieved successfully in all kinds of conditions, its use can be further expanded and can be used to achieve unimaginable and complex applications.

\section{REFERENCES}

1. Florian Schroff, Dmitry Kalenichenko and James Philbin. FaceNet: A Unified Embedding for Face Recognition and Clustering, arXiv: $1503.03832 v 3$, June 2015.

2. M. Monfort et al., "Moments in Time Dataset: One Million Videos for Event Understanding," in IEEE Transactions on Pattern Analysis and Machine Intelligence, vol. 42, no. 2, pp. 502-508, 1 Feb. 2020, doi: 10.1109/TPAMI.2019.2901464.

3. R. Singh, S. Pal., "Application of Machine Learning Algorithms to Predict Students Performance". International Journal of Advanced Science and Technology, Vol 29, No. 5, pp 7249-7261, 2020.

4. Ram Basnet, Srinivas Mukkamala, Andrew H Sung., "Detection of phishing attacks: A machine learning approach". In Soft Computing Applications in Industry, pages 373-383. Springer, 2008. 
5. R. Punnoose, P. Ajit., "Prediction of Employee Turnover in Organizations using Machine Learning Algorithms," Int. J. Adv. Res. Artif. Intell., vol. 5, no. 9, pp. 22-26, 2016, doi: 10.14569/ijarai.2016.050904.

6. Kaiming He Xiangyu Zhang Shaoqing Ren Jian Sun: Deep Residual Learning for Image Recognition, arXiv:1512.03385v1, 10 Dec 2015.

7. Sokolova M, Lapalme G: A systematic analysis of performance measures for classification tasks. Inform Process Manag 45: pp. 427-437, 2009.

8. Zhao, W., Chellappa, R., Krishnaswamy, A.: Discriminant Analysis of Principal Components for Face Recognition, Proc. of the 3rd IEEE International Conference on Face and Gesture Recognition, FG'98, (1998) 336.

9. Rathod, H.; Ware, Y.; Sane, S.; Raulo, S.; Pakhare, V.; Rizvi, I.A: Automated attendance system using machine learning approach. In Proceedings of the 2017 International Conference on Nascent Technologies in Engineering (ICNTE), Navi Mumbai, Maharashtra, India, 9-10 January 2017; pp. 1-5.

10. Rakesh Saini, Abhishek Saini, Deepak Agarwal: "Analysis of Different Face Recognition Algorithms", International Journal Of Engineering Research \& Technology (IJERT) Volume 03, Issue 11, November 2014.

11. Al Zorgani, M. and Ugail, H. Comparative Study of Image Classification using Machine Learning Algorithms, The 2nd Annual Innovative Engineering Research Conference (AIERC 2018), October 2018.

12. S. Balaban, "Deep learning and face recognition: The state of the art", Proc. SPIE, vol. 9457, May 2015.

13. Davis E King. Dlib-ml: A machine learning toolkit. The Journal of Machine Learning Research, 10:1755-1758, 2009.
14. Wei, X.Y.; Yang, Z.Q.: Mining in-class social networks for large-scale pedagogical analysis. In Proceedings of the 20th ACM international conference on Multimedia, Nara, Japan, 29 October-2 November 2012; pp. 639-648.

15. Dosovitskiy, J. T. Springenberg, and T. Brox. Unsupervised feature learning by augmenting single images, arXiv preprint arXiv:1312.5242, 2013.

16. LI.Masi, A. T. Tran, T. Hassner, J. T. Leksut, and G. Medioni, "Do we really need to collect millions of faces for effective face recognition?" in European Conference on Computer Vision, 2016.

17. Eko Setiawan, Adharul Muttaqin: Implementation of K-Nearest Neighbours Face Recognition on Low-power Processor, TELKOMNIKA, Vol.13, No.3, September 2015, pp. 949-954.

18. Emir Kremic and Abdulhamit Subasi: Performance of Random Forest and SVM in Face Recognition Performance of Random Forest and SVM in Face Recognition, The International Arab Journal of Information Technology, Vol. 13, NO. 2, March 2016.

19. N. A. A. Shashoa, N. A. Salem, I. N. Jleta and O. Abusaeeda, "Classification depend on linear discriminant analysis using desired outputs," 17th International Conference on Sciences and Techniques of Automatic Control and Computer Engineering (STA), Sousse, 2016, pp. 328-332, doi: 10.1109/STA.2016.7952041.

20. Amos, Brandon et al. "OpenFace: A general- purpose face recognition library with mobile applications, 2016. 